\title{
Simplifying Two Families of Nonlinear Ordinary Differential Equations
}

\author{
Feng Qi ${ }^{1,2,3, \dagger} \quad$ Jing-Lin Wang $^{3} \quad$ Bai-Ni Guo ${ }^{4}$ \\ ${ }^{1}$ Institute of Mathematics, Henan Polytechnic University, \\ Jiaozuo City, Henan Province, 454010, China \\ ${ }^{2}$ College of Mathematics, Inner Mongolia University for Nationalities, \\ Tongliao City, Inner Mongolia Autonomous Region, 028043, China \\ ${ }^{3}$ Department of Mathematics, College of Science, \\ Tianjin Polytechnic University, Tianjin City, 300387, China \\ ${ }^{4}$ School of Mathematics and Informatics, Henan Polytechnic University, \\ Jiaozuo City, Henan Province, 454010, China \\ ${ }^{\dagger}$ Corresponding author: qifeng618@gmail.com, qifeng618@hotmail.com
}

\begin{abstract}
In the paper, by virtue of techniques in combinatorial analysis, the authors simplify two families of nonlinear ordinary differential equations in terms of the Stirling numbers of the first kind.

2010 Mathematics Subject Classification: Primary 34A05; Secondary 11B37, 34A34.

Key words and phrases: nonlinear ordinary differential equation; Stirling number of the first kind; simplifying; technique; combinatorial analysis.
\end{abstract}

\section{Motivation and main results}

In [3, Theorem 1], it was acquired inductively and recursively that the nonlinear differential equations

$$
G^{(n)}(t)=\frac{(-1)^{n}(n-1) !}{(1+t)^{n}} \sum_{j=2}^{n+1}(j-1) ! H_{n-1, j-2} G^{j}(t)
$$

for $n \in \mathbb{N}$ have a solution $G(t)=\frac{1}{\ln (1+t)}$, where

$$
H_{n, 0}=1, \quad H_{n, 1}=H_{n}=\sum_{k=1}^{n} \frac{1}{k}, \quad \text { and } \quad H_{n, j}=\sum_{k=j}^{n} \frac{H_{k-1, j-1}}{k}
$$

for $2 \leq j \leq n$ and $n \in \mathbb{N}$. Thereafter, the identity 


$$
\begin{aligned}
&(-1)^{m} \sum_{j=0}^{\min \{n, m-1\}}(m-j) !(m-1) ! H_{m-1, m-j-1}\langle n\rangle_{j} b_{n-j}^{(m-j+1)} 0 \leq n \leq m \\
&= \begin{cases}(-1)^{m} m !\langle m\rangle_{n}, & \\
\sum_{\ell=0}^{n-m-1}\left(\begin{array}{c}
m \\
\ell
\end{array}\right) \frac{\langle n\rangle_{\ell+m+1}}{n-\ell} b_{n-\ell}, & n \geq m+1,\end{cases}
\end{aligned}
$$

for $m \in \mathbb{N}$ and $n \geq 0$ was derived in [3, Theorem 2], where the higher order Bernoulli numbers of the second kind $b_{n}^{(k)}$ can be generated by

$$
\left[\frac{t}{\ln (1+t)}\right]^{k}=\sum_{n=0}^{\infty} b_{n}^{(k)} \frac{t^{n}}{n !}
$$

with $b_{n}=b_{n}^{(1)}$, and the falling factorial $\langle x\rangle_{n}$ of $x \in \mathbb{R}$ is defined by

$$
\langle x\rangle_{n}=\prod_{k=0}^{n-1}(x-k)= \begin{cases}x(x-1) \cdots(x-n+1), & n \geq 1 \\ 1, & n=0\end{cases}
$$

In [4, Theorem 2.1], it was inductively and recursively obtained that the family of differential equations

$$
F^{(n)}(t)=\frac{(-1)^{n-1}}{(1+\lambda t)^{n}} \sum_{k=1}^{n}(k-1) ! \lambda^{n-k}(n-1) ! H_{n-1, k-1} e^{-k F(t)}, \quad n \in \mathbb{N}
$$

have a solution

$$
F(t)=\ln \left[1+\frac{\ln (1+\lambda t)}{\lambda}\right]
$$

As an application of the above result, the identity

$$
\begin{aligned}
&(-1)^{m+n+1} \frac{(m+n) !}{(m-1) !} \sum_{\ell=0}^{m+n+1} \frac{D_{\ell, \lambda}}{\ell !} \frac{(-\lambda)^{m+n-\ell-1}}{m+n-\ell}=\sum_{k=1}^{m} \sum_{m_{3}=0}^{n} \sum_{m_{2}=0}^{m_{3}} \sum_{m_{1}=0}^{m_{2}}(-1)^{m_{1}+m_{3}}\left(\begin{array}{c}
n \\
m_{3}
\end{array}\right) \\
& \times\left\langle m+n-m_{3}-1\right\rangle_{n-m_{3}}(k-1) ! k^{m_{1}} \lambda^{m+n-k-m_{2}} s\left(m_{2}, m_{1}\right) s\left(m_{3}, m_{2}\right) H_{m-1, k-1}
\end{aligned}
$$

for $m \in \mathbb{N}$ and $n \geq 0$ was derived in [4, Theorem 3.1], where $D_{n, \lambda}$ is generated by

$$
\frac{\lambda \ln \left[1+\frac{\ln (1+\lambda t)}{\lambda}\right]}{\ln (1+\lambda t)}=\sum_{n=0}^{\infty} D_{n, \lambda} \frac{t^{n}}{n !}
$$

and the Stirling numbers of the first kind $s(n, k)$ can be generated by

$$
\frac{[\ln (1+x)]^{k}}{k !}=\sum_{n=k}^{\infty} s(n, k) \frac{x^{n}}{n !}, \quad|x|<1 .
$$

In [6, Corollary 2.3], it was inductively and recursively procured that the Stirling numbers of the first kind $s(n, k)$ for $1 \leq k \leq n$ can be expressed as

$$
s(n, k)=(-1)^{n+k}(n-1) ! \sum_{\ell_{1}=1}^{n-1} \frac{1}{\ell_{1}} \sum_{\ell_{2}=1}^{\ell_{1}-1} \frac{1}{\ell_{2}} \cdots \sum_{\ell_{k-2}=1}^{\ell_{k-3}-1} \frac{1}{\ell_{k-2}} \sum_{\ell_{k-1}=1}^{\ell_{k-2}-1} \frac{1}{\ell_{k-1}} .
$$


See also [5, p. 27, Remark 3.3]. Comparing (2) with 4, we observe that

$$
(-1)^{n+k} s(n, k)=(n-1) ! H_{n-1, k-1}, \quad n \geq k \geq 1 .
$$

See also [12, pp. 7-8, Section 2.4] and [13, p. 9, Section 2.5].

By virtue of the identity (5), the above-mentioned Theorems 1 and 2 in [3] can be alternatively restated as the following theorem.

Theorem 1. The nonlinear differential equations

$$
G^{(n)}(t)=\frac{1}{(1+t)^{n}} \sum_{k=1}^{n}(-1)^{k} k ! s(n, k) G^{k+1}(t), \quad n \in \mathbb{N}
$$

have a solution $G(t)=\frac{1}{\ln (1+t)}$ and the identity

$$
\begin{aligned}
(-1)^{m} \sum_{j=0}^{\min \{n, m-1\}}(-1)^{j}(m-j) ! s(m, m-j)\langle n\rangle_{j} b_{n-j}^{(m-j+1)} & \\
& = \begin{cases}(-1)^{m} m !\langle m\rangle_{n}, & 0 \leq n \leq m \\
\sum_{\ell=0}^{n-m-1}\left(\begin{array}{c}
m \\
\ell
\end{array}\right) \frac{\langle n\rangle_{\ell+m+1}}{n-\ell} b_{n-\ell}, & n \geq m+1\end{cases}
\end{aligned}
$$

is valid for $m \in \mathbb{N}$ and $n \geq 0$.

By virtue of the identity (5) once again, the above-mentioned Theorems 2.1 and 3.1 in [4] can be simply reformulated as Theorem 2 below.

Theorem 2. The family of differential equations

$$
F^{(n)}(t)=\frac{1}{(1+\lambda t)^{n}} \sum_{k=1}^{n}(-1)^{k-1}(k-1) ! \lambda^{n-k} s(n, k) e^{-k F(t)}, \quad n \in \mathbb{N}
$$

have a solution

$$
F(t)=\ln \left[1+\frac{\ln (1+\lambda t)}{\lambda}\right]
$$

and the identity

$$
\begin{aligned}
& \sum_{\ell=0}^{m+n+1} \frac{(-1)^{\ell}}{\ell ! \lambda^{\ell+1}(m+n-\ell)} D_{\ell, \lambda}=\frac{(-1)^{m}}{(m+n) !} \sum_{k=1}^{m} \sum_{m_{3}=0}^{n} \sum_{m_{2}=0}^{m_{3}} \sum_{m_{1}=0}^{m_{2}} \frac{(-1)^{m_{1}+m_{3}+k}}{\lambda^{k+m_{2}}} \\
& \quad \times\left(\begin{array}{c}
n \\
m_{3}
\end{array}\right)\left\langle m+n-m_{3}-1\right\rangle_{n-m_{3}}(k-1) ! k^{m_{1}} s\left(m_{2}, m_{1}\right) s\left(m_{3}, m_{2}\right) s(m, k)
\end{aligned}
$$

is valid for $m \in \mathbb{N}$ and $n \geq 0$.

Although the method used in proofs of [3, Theorem 1] and [4, Theorem 2.1] is effectual, however, in our eyes, proofs of their main results in [3, Theorem 1] and [4, Theorem 2.1] are a little long and tedious (they spent six pages and five pages, respectively), and formulations of their main results are less meaningful and significant (the Stirling numbers of the first kind $s(n, k)$ are more meaningful and significant than the quantities $\left.H_{n, k}\right)$.

The aim of this paper is to simply and concisely verify Theorems 1 and 2 by virtue of techniques in combinatorial analysis. 


\section{Simple proofs of main results}

Now we are in a position to prove our main results.

Proof of Theorem 1. In combinatorial analysis, the Faà di Bruno formula plays an important role and can be described in terms of the Bell polynomials of the second kind

$$
\mathrm{B}_{n, k}\left(x_{1}, x_{2}, \ldots, x_{n-k+1}\right)=\sum_{\substack{1 \leq i \leq n, \ell_{i} \in\{0\} \cup \mathbb{N} \\ \sum_{i=1}^{n} i \ell_{i}=n \\ \sum_{i=1}^{n} \ell_{i}=k}} \frac{n !}{\prod_{i=1}^{n-k+1} \ell_{i} !} \prod_{i=1}^{n-k+1}\left(\frac{x_{i}}{i !}\right)^{\ell_{i}}
$$

for $n \geq k \geq 0$, see [1, p. 134, Theorem A], by

$$
\frac{\mathrm{d}^{n}}{\mathrm{~d} t^{n}}[f \circ h(t)]=\sum_{k=0}^{n} f^{(k)}(h(t)) \mathrm{B}_{n, k}\left(h^{\prime}(t), h^{\prime \prime}(t), \ldots, h^{(n-k+1)}(t)\right)
$$

for $n \geq 0$, see [1, p. 139, Theorem C]. Setting $f(u)=\frac{1}{u}$ and $u=h(t)=\ln (1+t)$ in 10 gives

$$
\begin{gathered}
G^{(n)}(t)=\sum_{k=0}^{n}\left(\frac{1}{u}\right)^{(k)} \mathrm{B}_{n, k}\left(\frac{1}{1+t},-\frac{1}{(1+t)^{2}}, \ldots,(-1)^{n-k} \frac{(n-k) !}{(1+t)^{n-k+1}}\right) \\
=\sum_{k=0}^{n} \frac{(-1)^{k} k !}{u^{k+1}}\left(\frac{1}{1+t}\right)^{n}(-1)^{n+k} \mathrm{~B}_{n, k}(0 !, 1 !, 2 !, \ldots,(n-k) !)=\frac{1}{(1+t)^{n}} \sum_{k=0}^{n} \frac{(-1)^{k} k !}{\ln ^{k+1}(1+t)} s(n, k) \\
=\frac{1}{(1+t)^{n}} \sum_{k=0}^{n}(-1)^{k} k ! s(n, k) G^{k+1}(t)=\frac{1}{(1+t)^{n}} \sum_{k=1}^{n}(-1)^{k} k ! s(n, k) G^{k+1}(t)
\end{gathered}
$$

for $n \in \mathbb{N}$, where we used in the above lines the formula $s(n, 0)=0$ for $n \in \mathbb{N}$ and the identities

$$
\mathrm{B}_{n, k}\left(a b x_{1}, a b^{2} x_{2}, \ldots, a b^{n-k+1} x_{n-k+1}\right)=a^{k} b^{n} \mathrm{~B}_{n, k}\left(x_{1}, x_{2}, \ldots, x_{n-k+1}\right)
$$

and

$$
\mathrm{B}_{n, k}(0 !, 1 !, 2 !, \ldots,(n-k) !)=(-1)^{n-k} s(n, k)
$$

listed in $[1$, p. 135 , Theorem B]. This means that the nonlinear differential equations (6) have a solution $G(t)=\frac{1}{\ln (1+t)}$.

The proof of the identity (7) follows from combining proofs of the equation (6) and [3, Theorem 2] together.

Proof of Theorem 2. Setting $f(u)=\ln (1+u)$ and $u=h(t)=\frac{\ln (1+\lambda t)}{\lambda}$ in (10) gives

$$
\begin{aligned}
F^{(n)}(t) & =\sum_{k=0}^{n}[\ln (1+u)]^{(k)} \mathrm{B}_{n, k}\left(\frac{1}{1+\lambda t},-\frac{\lambda}{(1+\lambda t)^{2}}, \ldots,(-1)^{n-k} \frac{(n-k) ! \lambda^{n-k}}{(1+\lambda t)^{n-k+1}}\right) \\
& =\sum_{k=0}^{n}[\ln (1+u)]^{(k)}\left(\frac{1}{1+\lambda t}\right)^{n}(-1)^{n+k} \lambda^{n-k} \mathrm{~B}_{n, k}(0 !, 1 !, 2 !, \ldots,(n-k) !)
\end{aligned}
$$




$$
\begin{aligned}
& =\left(\frac{1}{1+\lambda t}\right)^{n} \sum_{k=0}^{n}[\ln (1+u)]^{(k)}(-1)^{n+k} \lambda^{n-k}(-1)^{n-k} s(n, k) \\
& =\frac{1}{(1+\lambda t)^{n}}\left[\ln (1+u) \lambda^{n} s(n, 0)+\sum_{k=1}^{n} \frac{(-1)^{k-1}(k-1) !}{(1+u)^{k}} \lambda^{n-k} s(n, k)\right] \\
& =\frac{1}{(1+\lambda t)^{n}} \sum_{k=1}^{n} \frac{(-1)^{k-1}(k-1) !}{\left[1+\frac{\ln (1+\lambda t)}{\lambda}\right]^{k}} \lambda^{n-k} s(n, k) \\
& =\frac{1}{(1+\lambda t)^{n}} \sum_{k=1}^{n}(-1)^{k-1}(k-1) ! \lambda^{n-k} s(n, k) e^{-k F(t)}
\end{aligned}
$$

for $n \in \mathbb{N}$, where we used in the above lines the formula $s(n, 0)=0$ for $n \in \mathbb{N}$ and the identities (11) and (12). This means that the nonlinear differential equations 8 have a solution $F(t)=\ln 1+$ $\left.\frac{\ln (1+\lambda t)}{\lambda}\right]$.

The proof of the identity $(9)$ follows from combining proofs of the equation (8) and $[4$, Theorem 3.1] together.

\section{Remarks}

Finally we list several remarks on our main and the closely related results.

Remark 1 . The relation (5) shows that we should not call the quantities $H_{n, k}$ defined in (2) the generalized harmonic numbers, as did in [4, p. 745].

Remark 2. Till now we can see that formulations and proofs of Theorems 1 and 2 in this paper are simpler, more concise, more meaningful, and more significant than those in the papers [3, 4].

Remark 3. About simplification from the differential equations (1) and (3) to (6) and (8), the idea comes from the papers [2, 17, 8, 9, 10, 11, 12, 13, 14] and the closely related references therein.

\section{References}

[1] L. Comtet, Advanced Combinatorics: The Art of Finite and Infinite Expansions, Revised and Enlarged Edition, D. Reidel Publishing Co., Dordrecht and Boston, 1974.

[2] B.-N. Guo and F. Qi, Some identities and an explicit formula for Bernoulli and Stirling numbers, J. Comput. Appl. Math. 255 (2014), 568-579; Available online at http://dx.doi.org/ $10.1016 /$ j.cam.2013.06.020

[3] D. S. Kim and T. Kim, Some identities for Bernoulli numbers of the second kind arising from a non-linear differential equation, Bull. Korean Math. Soc. 52 (2015), no. 6, 2001-2010; Available online at http://dx.doi.org/10.4134/BKMS.2015.52.6.2001.

[4] T. Kim and D. S. Kim, Some identities of degenerate Daehee numbers arising from certain differential equations, J. Nonlinear Sci. Appl. 10 (2017), no. 2, 744-751; Available online at http://dx.doi.org/10.22436/jnsa.010.02.35. 
[5] F. Qi, Diagonal recurrence relations for the Stirling numbers of the first kind, Contrib. Discrete Math. 11 (2016), 22-30; Available online at http://hdl.handle.net/10515/sy5wh2dx6 and http://dx.doi.org/10515/sy5wh2dx6.

[6] F. Qi, Explicit formulas for computing Bernoulli numbers of the second kind and Stirling numbers of the first kind, Filomat 28 (2014), no. 2, 319-327; Available online at http:// dx.doi.org/10.2298/FIL14023190

[7] F. Qi, Explicit formulas for the convolved Fibonacci numbers, ResearchGate Working Paper (2016), available online at http://dx.doi.org/10.13140/RG.2.2.36768.17927.

[8] F. Qi and B.-N. Guo, Explicit formulas and nonlinear ODEs of generating functions for Eulerian polynomials, ResearchGate Working Paper (2016), available online at http://dx.doi . org/10.13140/RG.2.2.23503.69288

[9] F. Qi and B.-N. Guo, Explicit formulas for derangement numbers and their generating function, J. Nonlinear Funct. Anal. 2016, Article ID 45, 10 pages.

[10] F. Qi and B.-N. Guo, Some properties of a solution to a family of inhomogeneous linear ordinary differential equations, Preprints 2016, 2016110146, 11 pages; Available online at http://dx. doi.org/10.20944/preprints201611.0146.v1.

[11] F. Qi and B.-N. Guo, Some properties of the Hermite polynomials and their squares and generating functions, Preprints 2016, 2016110145, 14 pages; Available online at http://dx.doi. org/10.20944/preprints201611.0145.v1

[12] F. Qi and B.-N. Guo, Viewing some nonlinear ODEs and their solutions from the angle of derivative polynomials, ResearchGate Working Paper (2016), available online at http://dx. doi.org/10.13140/RG.2.1.4593.1285

[13] F. Qi and B.-N. Guo, Viewing some ordinary differential equations from the angle of derivative polynomials, Preprints 2016, 2016100043, 12 pages; Available online at http://dx.doi.org/ 10.20944/preprints201610.0043.v1

[14] F. Qi, J.-L. Zhao, and J.-L. Wang, Derivative polynomials of a function related to the ApostolEuler and Frobenius-Euler numbers, J. Nonlinear Sci. Appl. 10 (2017), in press. ResearchGate Working Paper (2017), available online at http://dx.doi.org/10.13140/RG.2.2.35572. 35204 .

(C) 2017 by the authors; licensee Preprints, Basel, Switzerland. This article is an open access article distributed under the terms and conditions of the Creative Commons Attribution (CC BY) license http://creativecommons.org/licenses/by/4.0/). 